\title{
Investigation of temporal bone asymmetry in cases with unilateral tinnitus: morphometric and multicentric clinical study
}

\author{
O.K. Kahveci ${ }^{1 \#}$, N. Gocmen-Mas ${ }^{2 \#}$, N. Okur ${ }^{3}$, M.D. Yılmaz ${ }^{4}$, O. Ozel ${ }^{5}$, F. Yucedaǧ ${ }^{1}$, \\ A.C. Yazici ${ }^{6}$ \\ ${ }^{1}$ Department of Otolaryngology, Afyon Kocatepe University, School of Medicine, Afyonkarahisar, Turkey \\ ${ }^{2}$ Department of Anatomy, Dokuz Eylul University School of Medicine, Izmir, Turkey \\ ${ }^{3}$ Department of Radiology, Afyon Kocatepe University, School of Medicine, Afyonkarahisar, Turkey \\ ${ }^{4}$ Antalya Training and Research Hospital Otolaryngology Clinic, Antalya, Turkey \\ ${ }^{5}$ Afyon State Hospital Otolaryngology Clinic, Afyonkarahisar, Turkey \\ ${ }^{6}$ Department of Biostatistics, Baskent University School of Medicine, Ankara, Turkey
}

[Received 13 August 2012; Accepted 24 September 2012]

\begin{abstract}
The aim of this multicentric study was carried out to compare the anatomical structures of temporal bones in patients with unilateral tinnitus in healthy ears. We also aimed at evaluating whether age- and gender-related asymmetrical changes occur in temporal bones or not. Fifty-two ears of 26 patients who had unilateral tinnitus were included into the retrospective study. The patients who had subjective nonpulsatile tinnitus and who previously had temporal computed tomography according to their file records were accepted to the study. Temporal computed tomography scans and audiometric results of patients were examined retrospectively. Middle ear volume, diameter of internal acoustic meats, and diameter of jugular bulb were evaluated by both anatomist and radiologist, in an interobserver manner. Internal acoustic meats and jugular bulb were found to be larger in the ears that had tinnitus than in the healthy ears; however, there was no statistical significance. The stereological morphometric study of the correlation with sex of temporal bone asymmetry in humans is of importance for both otolaryngologists and anatomists. These results will contribute to data on middle ear volume, internal acoustic meats, and jugular bulb sizes. (Folia Morphol 2013; 72, 1: 22-28)
\end{abstract}

Key words: unilateral tinnitus, temporal bone asymmetry, internal acoustic meats, jugular bulb volumetry, stereology

\section{INTRODUCTION}

Tinnitus is a commonly encountered complaint in patients of daily otolaryngology practice, but there is still little known about its underlying pathophysiology, aetiology, and effective treatment. Although pulsatile tinnitus can have numerous causes, it is easier for the clinician to find out the underlying pathology by radiological studies and treat tinnitus by surgical means [22]. It is challenging to find the underlying cause of non-pulsatile tinnitus, because of its subjective nature. Acoustic neuroma or otologic disease such as Meniere's disease can sometimes be the cause of non-pulsatile subjective tinnitus. On the other hand, the more common presen-

Address for correspondence: Associate Prof. N. Gocmen-Mas, Department of Anatomy, School of Medicine, Dokuz Eylul University, Izmir, Turkey, tel: +90 [232] 4124372, fax: +90 [232] 4129798, e-mail: nuketmas@gmail.com

\#The first two authors contributed equally to the work. 
tation is subjective tinnitus with hearing loss but without any neurologic, otologic disease or any constitutional lesion [28]. In this situation, possible theories are damaged hair cells of the inner ear and/or lack of suppression of activity in the auditory cortex, which can be caused by conditions such as acoustic trauma, oto-toxic drugs and head or ear trauma [3, 11, 27]. Some patients are more susceptible to tinnitus. Caucasians are affected by tinnitus more than African-Americans [27]. The factors that cause this susceptibility are unknown, but anatomical, physiological, and genetic factors may play a role. Genetic and physiological factors were investigated in a few studies $[9,27]$. However, there is no multicentric study that focuses on the morphometric ear differences and measurements of internal acoustic meats and also the jugular bulb in unilateral tinnitus patients in respect of gender, age, and asymmetry.

The aim of this study was to compare the anatomical structures of the temporal bone in patients with unilateral tinnitus with healthy ears with no tinnitus. Additionally, we aimed to evaluate whether age- and gender-related asymmetrical changes occur in temporal bones or not.

\section{MATERIALS AND METHODS}

\section{Clinical analysis}

Fifty-two ears of 26 patients with unilateral tinnitus who applied to the otolaryngology clinics of Kocatepe University, Antalya Training and Research Hospital and also Afyon Kocatepe State Hospital were included into the retrospective multicentric study. Patients who had subjective nonpulsatile tinnitus and previously had temporal bone computed tomography (CT) examination were accepted to the study according to the file records of the patients. Patients with otologic, neurologic, or systemic diseases and patients using ototoxic agents were excluded from the study. Patients who had objective and pulsatile tinnitus were not included in the study. Of the patients, 12 had right-sided tinnitus and 14 had left-sided tinnitus. Patients' contra lateral ears with no sensation of tinnitus were accepted as a healthy control group. The ears were divided into four groups as "Right tinnitus group", "Right healthy group", "Left tinnitus group", and "Left healthy group".

Temporal CT scans and audiometry results of patients were examined, retrospectively.

\section{Computed tomographic analysis}

The CT scans of 26 right handed patients who had unilateral tinnitus were included into the study according to file records. All evaluations were performed with a Siemens Samoten Emotion multi-slice CT machine (Germany). Patients who met the inclusion criteria underwent multi-detector row CT examination. Transverse scans were acquired in the helical mode with $120 \mathrm{mAs}$, 1-second rotation time, 1-mm section thickness, and 1-mm table feed and rotation. All images were displayed at a window centre of $700 \mathrm{HU}$ and a window width of $4000 \mathrm{HU}$. The retrospective morphometric study was approved by the Local Ethics Committee of Antalya Training and Research Hospital. According to archive file data, none of the participants was taking medication for 7 days or more at the time of scanning. The clinical evaluation ascertained both the personal and family history and physical and neurological conditions of the subjects by checking the patients' file records. Patients who had a lifetime history of neurological illness, migraine with aura, head injury, a history of any chronic medication use, substance or alcohol abuse, epilepsy, schizophrenia, bipolar disorder, major medical illnesses (including cardiac disease and diabetes) were excluded from the study.

\section{Morphometric and stereological analyses}

Middle ear volumes, diameters of internal acoustic meats, and diameters of the jugular bulb of each image were evaluated by anatomist and radiologist, in an interobserver manner. The diameters were measured three times manually using callipers for each of the CT images, bilaterally. The internal auditory canal is the indentation on the medial border of the petrous bone. The diameter of the internal auditory canal was measured at the internal opening of the acoustic canal in the petrous part of the temporal bone. The diameter of the jugular bulb was measured at its broadest section with the adjoining middle ear cavity (Figs.1-3). All measurements were performed blinded to subject details and data of any other results. An optimal plan was taken as the smallest size of anisotropic structures that may be measured in stereological volumetric analysis of axial temporal CT sections. Original CT images were exported as tiff image files, which were further subjected to stereological analysis. A uniform point-grid with a point-associated area of $0.625 \mathrm{~cm}^{2}$ was randomly superimposed on each CT image using the "Grid" plug-in installed with films. Points hitting the middle ears were manually counted for volume esti- 

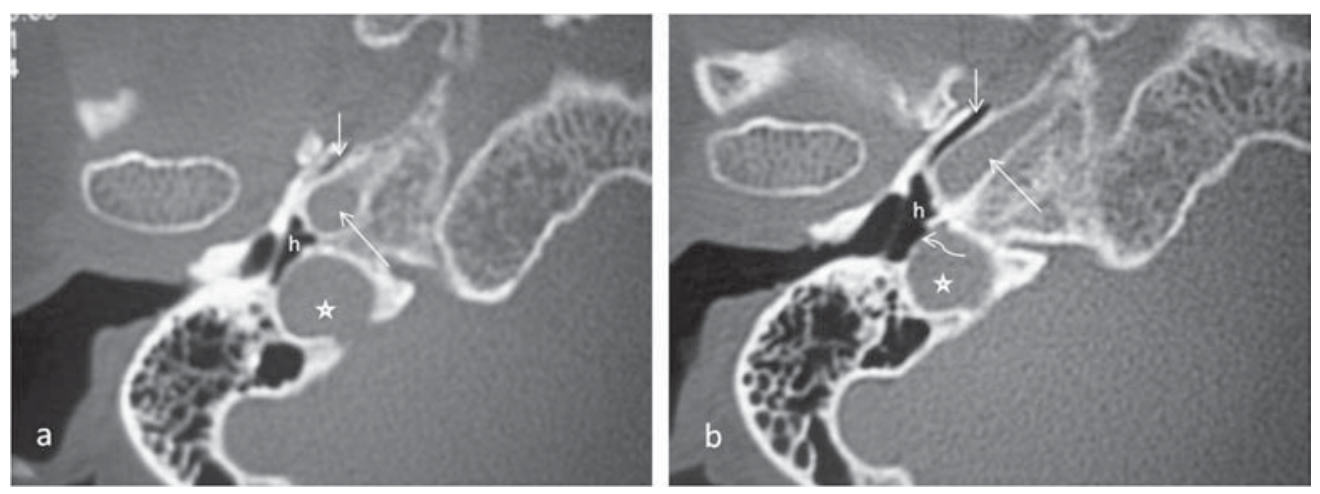

Figure 1. a. Transverse temporal computed tomography sections through the hypotympanum (h) in the caudocranial direction. Long arrow: carotid canal; short arrow: Eustachian tube; asterisk: jugular bulb. It is noted that the jugular bulb is partially dehiscent (b, curved arrow).
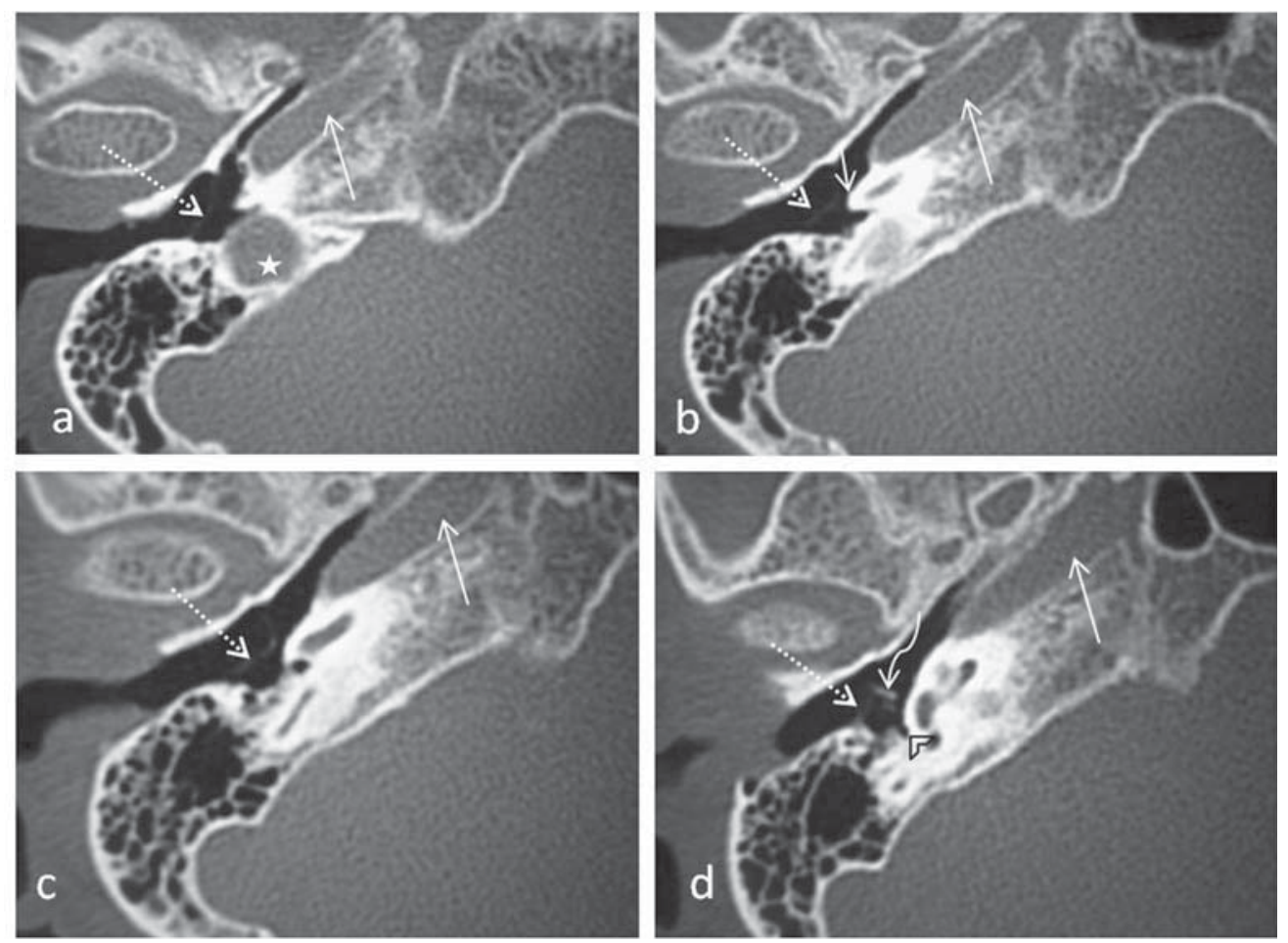

Figure 2a-d. Transvers temporal computed tomography sections through the mesotympanum in the caudocranial direction. Long arrow: carotid canal; short arrow: promontorium; asterisk: jugular bulb; dotted arrow: tympanic membrane; curved arrow: manubrium mallei; arrowhead: sinus tympani. Tympanic membrane shows a funnel-shaped appearance.

mation, bilaterally. The volumes were also estimated using the Cavalier's principle of stereological methods as described in previous studies $[1,10,12,15]$ by utilising the formula given below:

$$
\begin{aligned}
& V=t \times[(S U) \times d] / S L] 2 \times \Sigma P \\
& V=t \times[(S U) \times d] / S L] 2 \times \Sigma P
\end{aligned}
$$

Where: $\mathrm{t}$ is the section thickness, $\mathrm{SU}$ is the scale unit (the real length of the scale marked on the CTs), $\mathrm{d}$ is the distance between two points in the point grid, $\mathrm{SL}$ is the scale length (the actual measure of the scale on CTs), and P is the number of points counted. All data have been entered into a previously prepared Microsoft excel spreadsheet for automatic calculation of the outcomes of the above formula and the statistical evaluation parameters including the nugget variance and the coefficient of error (CE).

In order to evaluate volumetric asymmetry between right and left middle ears, middle sagittal sections were identified by clear visualisation of the area. The point counts belonging to the middle section were divided by two and the results were added to total point counts 

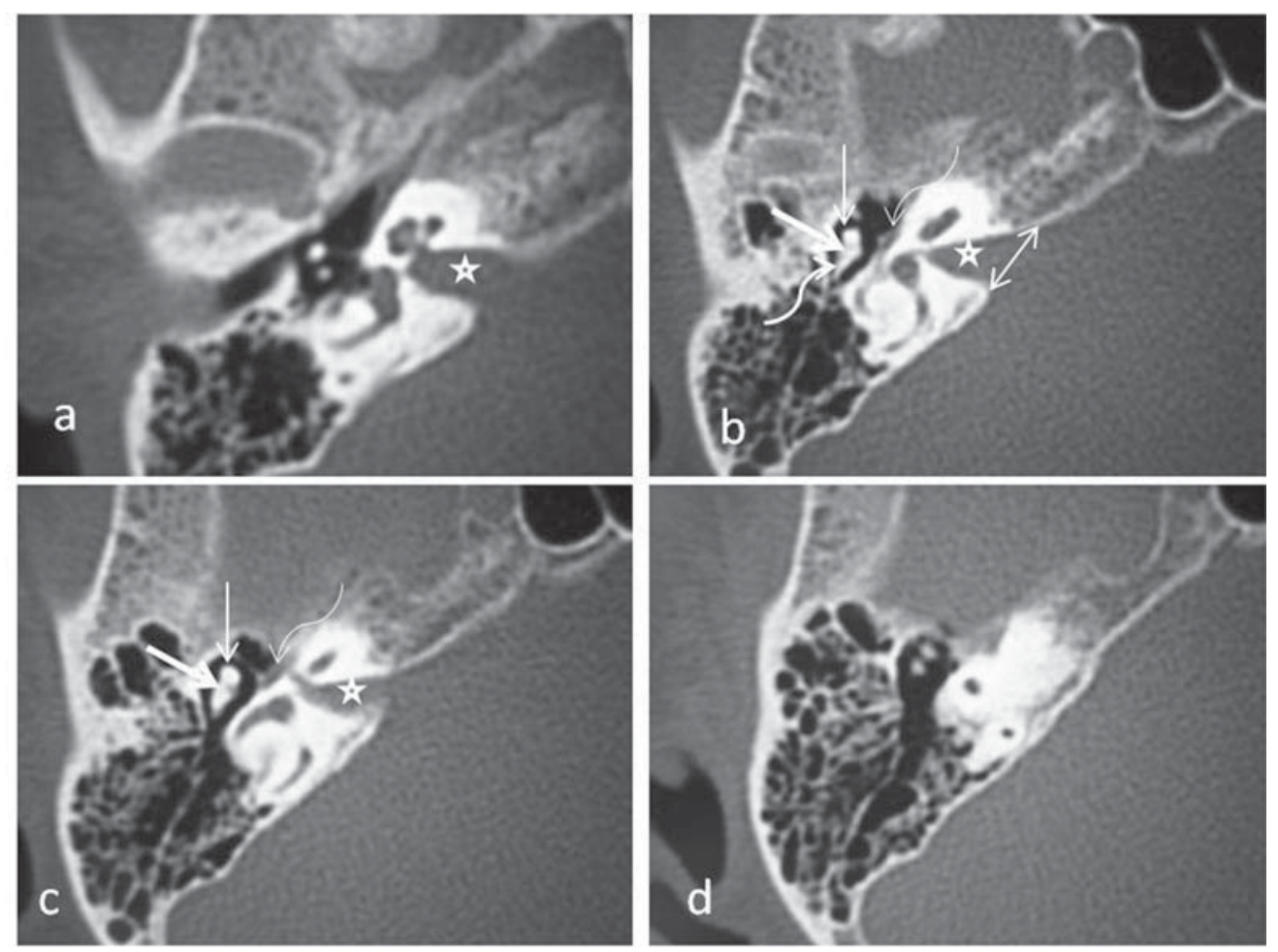

Figure 3a-d. Transvers temporal computed tomography sections through the epitympanum, which is the middle ear space superior to the uppermost aspect of the tympanic membrane. It houses the head of the malleus (b, $\mathbf{c}$, thin straight arrow), as well as the body (b, c, thick straight arrow) and short process (b, thick curved arrow) of the incus. Note the tympanic part of the facial nerve (b, c, thin curved arrow). The internal auditory canal (asterisk) is the indentation on the medial border of the petrous bone. The diameter of the internal auditory canal was measured at the internal opening of the acoustic canal in the petrous part of the temporal bone (b, double-headed arrow).

for each area separately, and thus a volume value for each middle ear was estimated. The stereological point counting method is demonstrated in Figure 4.

\section{Statistical analysis}

Shapiro-Wilk's test was used to assess the normality of distributions of the variables, and Levene's test was used to assess the homogeneity of variances in the different groups. Variables with normal distribution and homogeneous variance were analysed by Student's t test for comparison of the two independent groups and paired sample t test for dependent groups. Parametric test assumptions were not available for some variables, so the comparisons of these groups were performed with the Mann-Whitney $U$ test for two independent groups and the Wilcoxon signed rank test for dependent groups. The results of tests were expressed as the number of observations $(n)$, the mean \pm standard deviation, and median, (mean $\pm S D, M$ ). Data analyses were performed with SPSS 17.0 software (Statistical Package for the Social Sciences, version 17.0, SSPS Inc, and Chicago IL, USA). A p value of $<0.05$ was considered statistically significant.

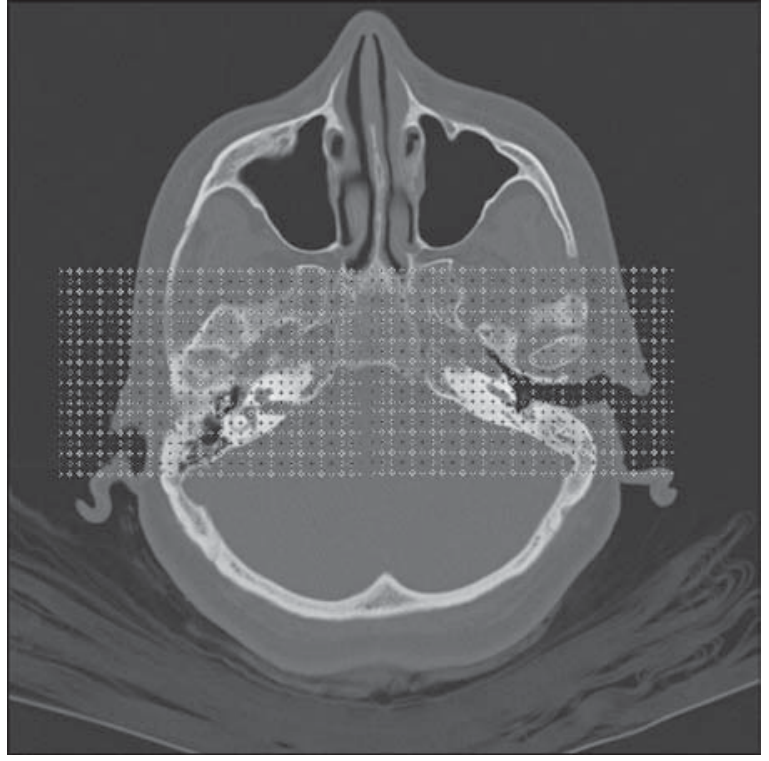

Figure 4. The stereological point counting method is shown on the axial computed tomography section of the temporal bone.

\section{RESULTS}

The mean age of patients was 52.12 years (range 24 to 70 years). There were 23 females and 3 males 
Table 1. Mean values of morphometric measurements according to sex groups

\begin{tabular}{lcccc}
\hline & $\begin{array}{c}\text { Right tinnitus } \\
\text { (mean } \pm \text { SD; median) }\end{array}$ & $\begin{array}{c}\text { Left healthy } \\
\text { (mean } \pm \text { SD; median) }\end{array}$ & $\begin{array}{c}\text { Left tinnitus } \\
\text { (mean } \pm \text { SD; median) }\end{array}$ & $\begin{array}{c}\text { Right healthy } \\
\text { (mean } \pm \text { SD; median) }\end{array}$ \\
\hline Middle ear volume $\left[\mathrm{cm}^{3}\right]$ & $\begin{array}{c}6.03 \pm 0.78 ; 5.56 \\
\mathrm{~N}=9\end{array}$ & $\begin{array}{c}6.24 \pm 0.57 ; 6.48 \\
\mathrm{~N}=9\end{array}$ & $6.05 \pm 1.04 ; 5.86$ & $6.24 \pm 1.36 ; 6.48$ \\
$\mathrm{~N}=11$ & $\mathrm{~N}=11$ \\
Internal acoustic meatus & $8.78 \pm 0.93 ; 8.85$ & $8.22 \pm 1.06 ; 7.95$ & $8.52 \pm 1.28 ; 8.50$ & $8.17 \pm 0.97 ; 8.30$ \\
diameter [mm] & $\mathrm{N}=10$ & $\mathrm{~N}=10$ & $\mathrm{~N}=11$ & $\mathrm{~N}=11$ \\
Jugular bulb diameter [mm] & $7.45 \pm 2.12 ; 7.95$ & $6.30 \pm 2.04 ; 7.00$ & $7.20 \pm 1.46 ; 7.50$ & $6.86 \pm 1.96 ; 6.50$ \\
& $\mathrm{~N}=6$ & $\mathrm{~N}=3$ & $\mathrm{~N}=7$ & $\mathrm{~N}=9$ \\
\hline
\end{tabular}

SD — standard deviation

Table 2. Comparison of ear groups ( $p$ values)

\begin{tabular}{lcccc}
\hline & $\begin{array}{c}\text { Right tinnitus }(\mathbf{n}=12) \\
\text { Left healthy }(\mathbf{n}=12)\end{array}$ & $\begin{array}{c}\text { Right tinnitus }(\mathbf{n}=12) \\
\text { Right healthy }(\mathbf{n}=14)\end{array}$ & $\begin{array}{c}\text { Left tinnitus }(\mathbf{n}=14) \\
\text { Right healthy }(\mathbf{n}=14)\end{array}$ & $\begin{array}{c}\text { Left tinnitus }(\mathbf{n}=14) \\
\text { Left healthy }(\mathbf{n}=12)\end{array}$ \\
\hline Middle ear volume & 0.512 & 0.674 & 0.419 & 0.673 \\
Internal acoustic meatus diameter & 0.131 & 0.179 & 0.276 & 0.376 \\
Jugular bulb diameter & 0.272 & 0.587 & 0.624 & 0.446 \\
\hline
\end{tabular}

in the study group. The mean duration of patients' complaint of tinnitus was $1.81 \pm 1.57$ years.

The mean pure tone thresholds were $19.44 \pm$ $\pm 14.06 \mathrm{~dB}$ for the ears with tinnitus and $15.7 \pm 10.87$ for the contra lateral healthy ears. There was no statistically significant difference between the ears $(p=0.260$ ). Of the patients, 6 had history of acoustic trauma. The mean pure tone thresholds of acoustic trauma patients was $18.32 \pm 12.6$ and they did not show any statistically significant difference from the other ears that had tinnitus $(p=0.441)$.

The mean morphometric and volumetric measurement values of ear groups according to sex were shown at Table 1. There was no difference between mean values of female and male ears in respect of asymmetrical volumetric changes in controls and the cases with tinnitus. Right tinnitus ears were compared with left healthy ears of the same patients, and also separately with right healthy ears of the patients who had tinnitus in their left ears. Likewise, left tinnitus ears were compared with right healthy ears of the same patients and also with left healthy ears of patients who had tinnitus in their right ears. Comparison results of ear groups are shown in Table 2. Diameters of internal acoustic meats and jugular bulbs were found to be bigger than in healthy ears. Both right and left tinnitus ears had greater diameter than both right and left healthy ears; however, there was no statistical significance when comparing groups.

\section{DISCUSSION}

Tinnitus is a very common complaint of patients in otolaryngology practice but there is little known about underlying pathophysiology, aetiology, and effective treatment. It is challenging to find the underlying cause of non-pulsatile tinnitus because of its subjective nature. Acoustic neuroma or otologic diseases such as Meniere's disease can sometimes be the cause of nonpulsatile subjective tinnitus $[5,6]$.

Asymmetrical and volumetric changes in intracranial structures are of importance for both clinicians and anatomists $[12,25]$.

In literature, there is limited data on middle ear volumes of both healthy and unhealthy subjects. Osborn et al. [24] examined middle ear volume in patients with aural atresia and investigated the role of middle ear volume as an adjunct measure in determining surgical candidacy. According to their data, the average age of patients at the time CT examination was 4.7 years. The average middle ear volume of the atretic ears was $0.34 \mathrm{cc}$ compared to an average of $0.51 \mathrm{cc}$ for non-atretic ears. The mean ratio of atretic to non-atretic volume was 0.67 . In patients who underwent serial scans, no statistically significant difference in rates of growth existed between atretic and non-atretic ears. They concluded that measurements of middle ear volume correlated well with clinical grading scales. Swarts et al. [30] aimed at evaluating the relationship between mastoid air cell surface area and volume of the mastoid air cell system in adult humans. 
There is limited volumetric data regarding the middle ear related with tinnitus in literature. The stereological volumetric evaluation on the tympanic cavity was presented on a project (2007), an abstract book (2007), a presentation (2007), and also as the manuscript (2010) on cases with tinnitus which by Kurkcuoglu et al. [18-21]. According to the results there was no statistically significant difference between the sexes for tympanic cavity volume measurements and between both sites of the same individuals on CT. Differ from the declarations, our study group evaluated bilateral middle ear volumes of patients who suffered from unilateral tinnitus and compared them with healthy subjects via stereological techniques. The resuls of our control group results were similar to those of Kurkcuoglu et al. [21]. Additionally, we also measured the sizes of the internal acoustic meats and the jugular bulb. According to our data, the measurements were larger in the ears that had tinnitus than in the healthy ears.

Quantitative analysis of CT imaging may improve its diagnostic progress and has the potential evidence to reveal the aetiology of diseases. However, there is some diversity on defining the volumetric analysis methods of intracranial structures or substructures in the literature, which are frequently named as "traced", "automatic" (atlas-based), "planimetry", "three-dimensional", "stereological", etc. Many authors claimed that the automatic method is fast, efficient, unbiased, and safe. But others noted the traced measurement method as the "gold standard" against the automatic method [10,12].

In contract to the previous studies, we used the stereological measurement technique, which was firstly used in the evaluation of cerebellar asymmetry by Gocmen-Mas et al. [12]. The stereological method is simple, cheap, reliable, and unbiased. Additionally, the point counting approach takes less time [10, 25]. Multiple hypotheses were proposed to explain the pathophysiology of tinnitus, yet none of these hypotheses were enough to elucidate the underlying mechanism perfectly. This may be because of the multi-aetiological nature of tinnitus. Otologic disorders, neurologic disease, metabolic disorders such as vitamin B12 deficiency, zinc deficiency and anaemia, ototoxic drugs, iatrogenic causes, and psychogenic disorders can cause subjective nonpulsatile tinnitus [27, 29]. Personal factors are also important for tinnitus. Perception and response of patients to tinnitus may vary. Under the same conditions some of the patients may complain from tinnitus much more than other patients. For example most police officers and soldiers are exposed to acoustic trauma but only some of them complain about tinnitus. It was suggested that there are two components of reporting tinnitus by patients: first, the necessary physiological or pathological conditions to provide a stimulus to the auditory cortex, and second the personal tendency to report a tinnitus as present [31]. There is a relation between personality and perception of tinnitus.

Middle ear aeration is critical for ossicular coupling and normal hearing [23]. Haginomori et al. [13] showed that after canal wall mastoidectomy procedure, middle ear aeration was closely related to hearing outcomes. Good or normal hearing is an essential factor for providing a stimulus to the auditory cortex, which is the first step to constitute tinnitus. Therefore, we assume that middle ear volume changes might play role in the underlying tinnitus mechanism.

The functional anatomy (i.e. tonotopy) of the human cochlear nerve is not known well. Disorders of the cochlear nerve and internal acoustic meats may cause tinnitus. Acoustic neuroma is a wellknown cause of tinnitus. Hamartomas of internal [4], vascular loops [14] and osteomas [7] of internal acoustic meats were reported as a cause of tinnitus. De Ridder et al. [8] showed that decompression of the vestibulocochlear can be effective in treating unilateral tinnitus. The gold standard technique for diagnosis of acoustic neuroma is magnetic resonance imaging [6]. Internal acoustic canal tumours usually enlarge the acoustic canal; therefore, CT also is an efficient tool for the diagnosis of acoustic tumours by showing this enlargement.

Venous aetiologies have been recognised as potential sources of tinnitus. Venous sinus dominance, transverse sinus stenosis, and venous diverticulum are often presented in clinical series of tinnitus. Jugular bulb abnormalities such as high location, dehiscence, and diverticula were reported as venous causes of pulsatile tinnitus (Sismanis pulsatile tinnitus) [17].

Hearing loss in tinnitus patients is also a subject of interest among researchers. Some of the tinnitus patients may have acoustic trauma findings in their audiometric examinations. On the other hand, many patients present with normal audiogram. In our study, the affected ears showed worse hearing thresholds than healthy ears, but it was not statistically significant. Auditory brain stem responses of tinnitus patients with normal audiograms were examined and found to have reduced amplitudes of wave I [26]. They suggested that even if the audiogram of patients is normal, there is hidden hearing loss in tinnitus patients. The authors were also found to be reduced in tinnitus patients with 
normal hearing [2]. Kim et al. [16] investigated extended high frequency (above $8 \mathrm{kHz}$ ) hearing loss in patients with normal audiograms, and $74 \%$ of the patients had hearing loss above $8 \mathrm{kHz}$.

\section{CONCLUSIONS}

As a conclusion, we investigated possible anatomical differences and asymmetry in temporal bones of tinnitus patients. We found that the diameters of internal acoustic meats and jugular bulb were larger in the ears of those with tinnitus than in those with healthy ears; however, there was no statistical significance. The method is simple, reliable, unbiased, and cheap. Further studies are required with larger samples correlating with the aging process in order to support these results.

\section{ACKNOWLEDGEMENTS}

The authors would like to thank Asc Prof. Sinan Canan for his kind support and assistance on the stereological methodology.

\section{REFERENCES}

1. Acer N, Sahin B, Usanmaz M, Tatoðlu H, Irmak Z (2008) Comparison of point counting and planimetry methods for the assessment of cerebellar volume in human using magnetic resonance imaging: a stereological study. Surg Radiol Anat, 30: 335-339.

2. Ami M, Abdullah A, Awang MA, Liyab B, Saim L (2008) Relation of distortion product otoacoustic emission with tinnitus. Laryngoscope, 118: 712-717.

3. Bauer CA (2004) Mechanisms of tinnitus generation. Curr Opin Otolaryngol Head Neck Surg, 12: 413-417.

4. Carvalho GA, Matthies C, Osorio E, Samii M (2003) Hamartomas of the internal auditory canal: report of two cases. Neurosurgery, 52: 944-948.

5. Crummer RW, Hassan GA (2004) Diagnostic approach to tinnitus. Am Fam Physician, 69:120-126.

6. Curtin HD, Hirsch WUr (2008) Imaging of acoustic neuromas. Neurosurg Clin N Am, 19:175-205.

7. Davis TC, Thedinger BA, Greene GM ( 2000) Osteomas of the internal auditory canal: a report of two cases. Am J Otol, 21: 852-856.

8. De Ridder D, Ryu H, Moller AR, Nowé V, Van de Heyning P, Verlooy J (2004) Functional anatomy of the human cochlear nerve and its role in microvascular decompressions for tinnitus. Neurosurgery, 54: 381-388.

9. Deniz M, Bayazit YA, Celenk F, Karabulut H, Yilmaz A, Gunduz B, Saridogan C, Dagli M, Erdal E, Menevse A (2010) Significance of serotonin transporter gene polymorphism in tinnitus. Otol Neurotol, 31: 19-24.

10. Ekinci N, Acer N, Akkaya A, Sankur S, Kabadayi T, Sahin B (2008) Volumetric evaluation of the relations among the cerebrum, cerebellum and brain stem in young subjects: a combination of stereology and magnetic resonance imaging. Surg Radiol Anat, 30: 489-494.

11. Fortune DS, Haynes DS, Hall JW (1999) Tinnitus. Current evaluation and management. Med Clin North Am, 83: 153-162.

12. Gocmen-Mas N, Pelin C, Canan S, Yazici AC, Zagyapan R, Senan S, Karabekir HS, Sahin B (2009) Stereological evalu- ation of volumetric asymmetry in healthy human cerebellum. Surg Radiol Anat, 31: 177-181.

13. Haginomori S, Takamaki A, Nonaka R, Mineharu A, Kanazawa A, Takenaka H (2009) Postoperative aeration in the middle ear and hearing outcome after canal wall down tympanoplasty with soft-wall reconstruction for cholesteatoma. Otol Neurotol, 30: 478-483.

14. Herzog JA, Bailey S, Meyer J (1997) Vascular loops of the internal auditory canal: a diagnostic dilemma. Am J Otol, 18: 26-31.

15. Kalkan E, Cander B, Gul M, Girisgin S, Karabagli H, Sahin B (2007) Prediction of prognosis in patients with epidural hematoma by a new stereological method. Tohoku J Exp Med, 211: 235-242.

16. Kim DK, Park SN, Kim HM, Son HR, Kim NG, Park KH, Yeo SW (2011) Prevalence and significance of high-frequency hearing loss in subjectively normal-hearing patients with tinnitus. Ann Otol Rhinol Laryngol, 120: 523-528.

17. Krishnan A, Mattox DE, Fountain AJ, Hudgins PA (2006) CT arteriography and venography in pulsatile tinnitus: preliminary results. Am J Neuroradiol, 27: 1635-1638.

18. Kurkcuoglu A, Kurkcuoglu S, Pelin C, Tuncer U, Mas N, Zagyapan R (2007) Volumetric evaluation of Tympanic cavity in the patients with Tinnitus: a stereological study. European Anatomy Congress, Sep, Praque.

19. Kurkcuoglu A, Kurkcuoglu S, Pelin C, Tuncer U, Mas N, Zagyapan (2007) Volumetric evaluation of Tympanic cavity in the patients with Tinnitus: a stereological study. (S3-P121) Surg Radiol Anat, 29: 511-526

20. Kurkcuoglu A, Pelin C, Zagyapan R, Mas N, Kurkcuoglu S (2007) Volumetric evaluation of Tympanic cavity in the patients with Tinnitus. Baskent Univeristy Scientific Research Project KA 07/60, Ankara.

21. Kurkcuoglu A, Kurkcuoglu SS, Inancli HM, Enoz M, Pelin C, Zagyapan R (2010) Measurement of tympanic cavity volume by the Cavalieri principle in Turkish population. Kulak Burun Bogaz Ihtis Derg, 20: 137-141.

22. Mehall CJ, Wilner HI, LaRouere MJ (1995) Pulsatile tinnitus associated with a laterally placed sigmoid sinus. Am J Neuroradiol, 16: 905-907.

23. Merchant SN, Ravicz ME, Puria S, Voss SE, Whittemore KR Jr, Peake WT, Rosowski JJ (1997) Analysis of middle ear mechanics and application to diseased and reconstructed ears. Am J Otol, 18: 139-154.

24. Osborn AJ, Oghalai JS, Vrabec JT (2011) Middle ear volume as an adjunct measure in congenital aural atresia. Int J Pediatr Otorhinolaryngol, 75: 910-914.

25. Roberts N, Puddephat MJ, McNulty V (2000) The benefit of stereology for quantitative radiology. Br J Radiol, 73: 679-697.

26. Schaette R, McAlpine D (2011) Tinnitus with a normal audiogram: physiological evidence for hidden hearing loss and computational model. J Neurosci, 31: 13452-13457.

27. Seidman MD, Standring RT, Dornhoffer JL ( 2010) Tinnitus: current understanding and contemporary management. Curr Opin Otolaryngol Head Neck Surg, 18: 363-368.

28. Shiley SG, Folmer RL, McMenomey SO (2005) Tinnitus and hyperacusis. In: Cummings CW, Flint PW, Haughey BH, Robbins KT, Thomas JR, Harker LA eds. Otolaryngology - head and neck surgery. Mosby Year Book, St. Louis, pp. 2832-2847.

29. Sismanis A (2011) Pulsatile Tinnitus: contemporary assessment and management. Curr Opin Otolaryngol Head Neck Surg, 19: 348-357.

30. Swarts JD, Doyle BM, Doyle WJ (2011) Relationship between surface area and volume of the mastoid air cell system in adult humans. J Laryngol Otol, 5: 1-5.

31. Welch D, Dawes PJ (2008) Personality and perception of tinnitus. Ear Hear, 29: 684-692. 